\title{
HREM OF GENERAL AND TWIST GRAIN BOUNDARIES*
}

K. L. Merkle and L. J. Thompson

Materials Science Division

Argonne National Laboratory

9700 S. Cass Ave.

Argonne, IL 60439

February 1999

The submitted manuscript has been created
by the University of Chicago as Operator of
Argonne National" Laboratory "Argonne"
under Contract No. W-31-109-ENG-38 with
the U.S. Department of Energy. The U.S.
Government retains for itself, and others
acting on its behalf, a paid-up, non
exclusive, irrevocable worldwide license in
said article to reproduce, prepare derivative
works, distribute copies to and display
publicly, by or on behalf of the Government.

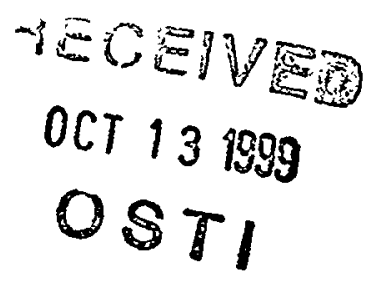

Paper to be submitted to Microscopy and Microanalysis, Portland, OR, August 1-5, 1999.

"Work supported by the U.S. Department of Energy, Basic Energy Sciences-Materials Sciences, grant No. W-31-109-ENG-38. 


\section{DISCLAIMER}

This report was prepared as an account of work sponsored by an agency of the United States Government. Neither the United States Government nor any agency thereof, nor any of their employees, make any. warranty, express or implied, or assumes any legal liability or responsibility for the accuracy, completeness, or usefulness of any information, apparatus, product, or process disclosed, or represents that its use would not infringe privately owned rights. Reference herein to any specific commercial product, process, or service by trade name, trademark, manufacturer, or otherwise does not necessarily constitute or imply its endorsement, recommendation, or favoring by the United States Government or any agency thereof. The views and opinions of authors expressed herein do not necessarily state or reflect those of the United States Government or any agency thereof. 


\section{DISCLAIMER}

Portions of this document may be illegible in electronic image products. Images are produced from the best available original document. 


\title{
HREM OF GENERAL AND TWIST GRAIN BOUNDARIES
}

\author{
K. L. Merkle and L. J. Thompson \\ Materials Science Division, Argonne National Laboratory, Argonne, ㅍ 60439
}

The observation of atomic-scale structures of grain boundaries (GBs) via axial illumination HREM has been largely restricted to tilt GBs, due to the requirement that the electron beam be parallel to a low-index zone axis on both sides of the interface. This condition can be fulfilled for all tilt GBs with misorientation about a low-index direction. The information obtained through HREM studies in many materials has brought important insights concerning the atomic-scale structure of such boundaries. However, it is well known that tilt GBs occupy only an infinitesimally small fraction of the 5-dimensional phase space which describes the macroscopic geometry of all GBs. Therefore, although tilt GBs are very important due to their low energy, it would be useful to also study twist GBs and general GBs that contain twist and tilt components.

We have prepared thin-film Au samples by an epitaxy technique in which (011) and (001) grains are grown side by side ${ }^{1}$. The resulting grain boundaries are general GBs, except for special geometries for which edge-on pure twist or tilt GBs can be produced. Briefly, an (011) Au film is physically transferred onto a (001) $\mathrm{NaCl}$ substrate at an in-plane rotation $\theta$ between the [100] directions in the film and the substrate. Low energy ion-beam sputtering and physical masking is used to remove most of the film, except for some seed crystals. Subsequently a film of the desired bicrystal geometry is grown by e-beam evaporation and equilibrated near $300^{\circ} \mathrm{C}$. Samples suitable for HREM are obtained by standard ion-milling techniques. HREM observation of GBs along [001] and [011] is possible as a function of $\theta$ as well as the inclination of the GB plane. The technique can be applied to any pair of zone-axis orientations for which epitaxy is achieved in the material investigated.

As an example, a (100) symmetric twist GB with misorientation angle $\Psi=45^{\circ}$ is shown in Fig. 1 . The HREM image was recorded utilizing a 4000EX JEOL microscope at a beam voltage of $400 \mathrm{kV}$ and a defocus value near $-65 \mathrm{~nm}$. Under the imaging used conditions both sides of the GB display white contrast features at the location of the atomic columns. The atomic height ledge is indicative of the migration mechanism for this GB. A film with the $\Psi=90^{\circ}$, (110) type symmetric twist GB is expected to be formed at facets that are bounded by the $(220)$ and $(0,2,-2)$ planes in the two grains respectively. However, Fig. 2 shows that this boundary, in contrast to the (100) twist GB does not remain planar, but reconstructs into microfacets that are bounded by (100) and (111) planes. Approximately every second (111) plane is coherently connected to every third (100) plane across the GB. We believe that this GB reconstructs, because the planar configuration has an extremely high energy. Atomistic computer simulations indicate that high-angle (110) periodic twist GBs have the highest GB energies and extremely disordered structures ${ }^{2}$.

Examples for general GBs, with tilt and twist components, are shown in Figs. 3 and 4. Although all of these GBs are incommensurate, distinct, almost identical, repeat structures can be recognized. These structural features are due to misfit localization, which, for example, occur in Fig. 3 when two (200) planes meet with one (111) plane in the upper crystal. The investigations show that general GBs are well structured at the atomic scale and often display features that are related to tilt GB structures, such as the tendency for coherence of lattice planes across the GB, misfit localization, as well as the formation of low-index facets (Fig. 4).

1. K. L. Merkle, J. Phys. Chem. Solids 55 (1994) 991.

2. D. Wolf and K. L. Merkle, in MATERIALS INTERFACES Atomic-level structure and properties, edited by D. Wolf and S. Yip (Chapman \& Hall, London, 1992) 87.

3. The authors acknowledge the use of the facilities of the ANL/MSD Electron Microscopy Center. This work was supported by the U.S. DOE, BES, under contract W-31-109-Eng-38. 


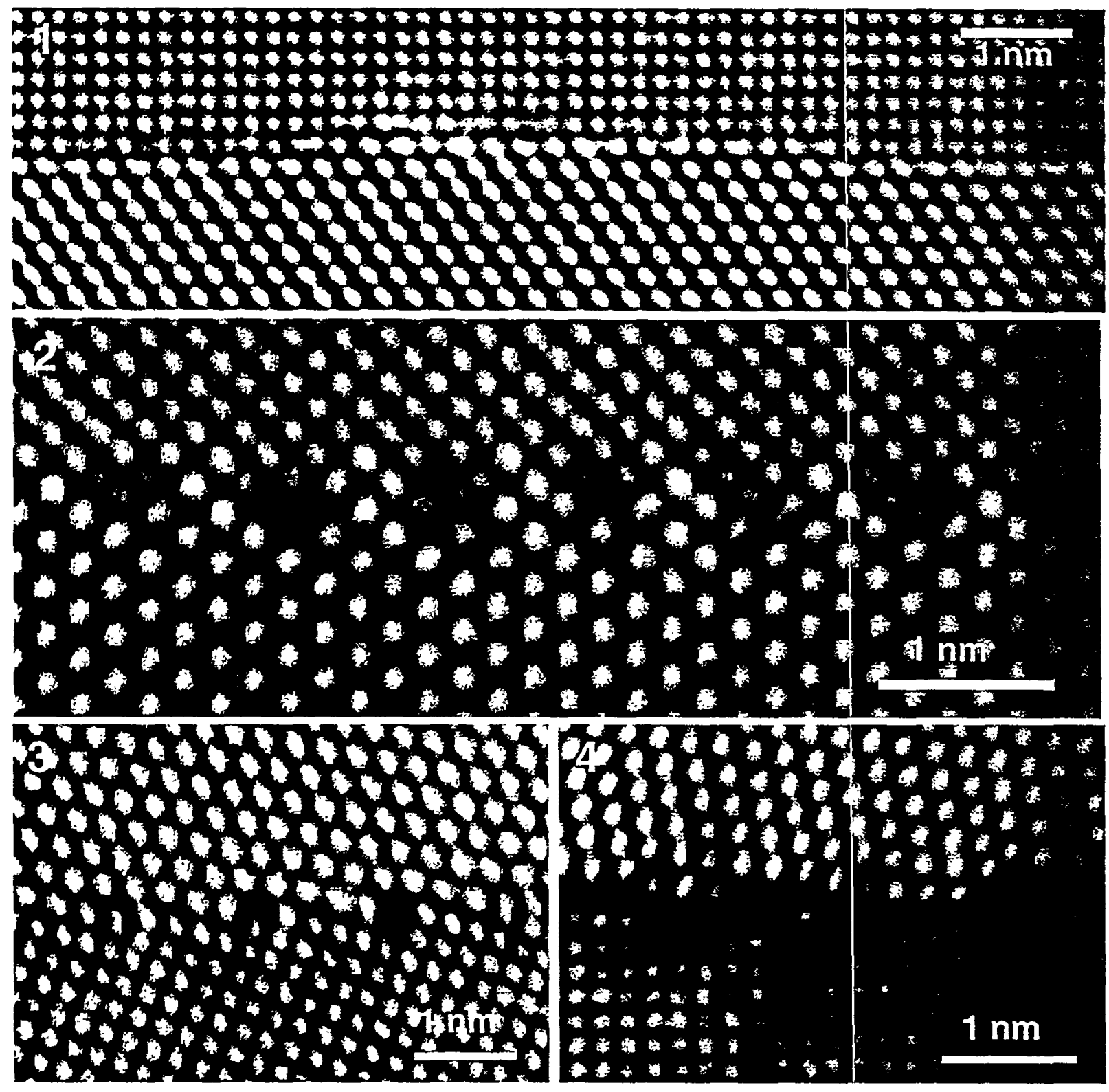

Fig. 1. HREM image of $\psi=45^{\circ},(010)$ symmetric twist GB, viewed along [001]. Note ledge and structural disorder in atomic planes next to the grain boundary.

Fig. 2. HREM image of $\psi=90^{\circ}$, (110) symmetric twist GB. The grain boundary does not remain planar, but reconstructs into mixed facets bounded by (111) and (200) planes.

Fig.3. Asymmetric (233)(250) grain boundary with tilt and twist components. Note almost identical repeat structures characterized by misfit localizations.

Fig 4. HREM image of (755)(100) grain boundary with tilt and twist components. Strain fields are causing image contrast effects at structural modulations. 